\title{
Joint Impact of Rain and Incidents on Traffic Stream Speeds
}

\author{
Mohammed Elhenawy $\mathbb{D}^{1},{ }^{1}$ Hesham A. Rakha $\mathbb{D}^{2},{ }^{2}$ and Huthaifa I. Ashqar $\mathbb{D}^{3}$ \\ ${ }^{1}$ Centre for Accident Research and Road Safety, Queensland University of Technology, Brisbane, Australia \\ ${ }^{2}$ Department of Civil and Environmental Engineering, Virginia Tech, Virginia Tech Transportation Institute, \\ 3500 Transportation Research Plaza, Blacksburg, VA 24061, USA \\ ${ }^{3}$ Booz Allen Hamilton, Washington, DC., USA \\ Correspondence should be addressed to Hesham A. Rakha; hrakha@vt.edu
}

Received 20 May 2020; Revised 14 December 2020; Accepted 21 December 2020; Published 11 January 2021

Academic Editor: Victor L. Knoop

Copyright (C) 2021 Mohammed Elhenawy et al. This is an open access article distributed under the Creative Commons Attribution License, which permits unrestricted use, distribution, and reproduction in any medium, provided the original work is properly cited.

\begin{abstract}
Unpredictable and heterogeneous weather conditions and road incidents are common factors that impact highway traffic speeds. A better understanding of the interplay of different factors that affect roadway traffic speeds is essential for policymakers to mitigate congestion and improve road safety. This study investigates the effect of precipitation and incidents on the speed of traffic in the eastbound direction of I-64 in Virginia. To the best of our knowledge, this is the first study that studies the relationship between precipitation and incidents as factors that would have a combined effect on traffic stream speeds. Furthermore, using a mixture model of two linear regressions, we were able to model the two different regimes that the traffic speed could be classified into, namely, free-flow and congested. Using INRIX traffic data from 2013 through 2016 along a 25.6-mi section of Interstate 64 in Virginia, results show that the reduction of traffic speed only due to incidents ranges from $41 \%$ to $75 \%$ if the road is already congested. In this case, precipitation was found to be statistically insignificant. However, regardless of the incident impact, the effect of light rain in free-flow conditions ranges from insignificant to a $4 \%$ speed reduction while the effect of heavy rain ranges from a $0.6 \%$ to a $6.5 \%$ speed reduction when the incident severity is low but has a roughly double effect when the incident severity is high.
\end{abstract}

\section{Introduction}

Unclear weather conditions and incidents are common factors that impact traffic speed. While weather conditions may include rain, snow, wind, and fog, traffic incidents are defined as any nonrecurring event that causes lane closures such as vehicle crashes, road construction, and work zones. With the increase in travel demand and number of vehicles, congestion has amplified impairing the efficiency of the road network, especially highways. To alleviate this consequence, developing novel Intelligent Transportation System (ITS) technologies and reforming policies for traffic management and operations has become substantial. By the same token, quantifying the extent of weather conditions and incidents on traffic stream speeds is a key in effective and real-time traffic management and operation. Understanding the factors that impact the traffic speed of road networks has attracted many researchers as it has a wide range of traffic planning, management, and operations related applications. This includes many autonomous vehicle technologies $[1,2]$, congestion mitigation and control [3], ecorouting technologies and vehicle routing operations [4], urban road management [5], public transit operations [6], travel time prediction $[7,8]$, and highway capacity analysis and prediction [9].

Studying the factors that impact the traffic is not a trivial task due to the complexity and highly dynamic nature of traffic and road conditions. On the one hand, weather is an important factor that impacts traffic stream speeds, but also causes $15 \%$ of all nonrecurring congestion in the United States [10]. Due to this, various studies have found that weather conditions, especially intensity of precipitation, have an important impact on traffic safety, demand, intensity, and flow $[11,12]$. The heterogeneity and relative 
unpredictability of precipitation in different traffic locations made understanding its effect appealing and important to policymakers. As a result, minimizing the precipitation impact on traffic operations as a traffic management strategy should consider the traffic volume of the road network and the behavior of drivers as they respond to different intensities of precipitation $[12,13]$. On the other hand, traffic incidents, which produce lane restrictions, work zone construction, and traffic accidents, are also one of the most important factors and tend to dramatically impact traffic for varying time periods [14]. Nevertheless, the rate of occurrence of these incidents implies that their cumulative impact on the traffic cannot be neglected when modeling and predicting traffic speed, as they cause about $35 \%$ of all nonrecurring congestion in the United States if we combined it with the work zone portion [10]. As a result, novel traffic management strategies to mitigate the effect of traffic incidents are essential to incident management agencies around the world.

\section{Literature Review}

Several previous studies attempted to analyze the effect of weather conditions and different incidents on the traffic stream speed in different locations. Weather conditions, especially rain, have a varying yet significant impact on traffic speed and its related traffic parameters, such as free-flow speed, traffic volume, and capacity depending on the road type and the severity of the situation, which trigger different responses by the drivers [15]. Ibrahim and Hall investigated the effect in two different rain severity [12]. They found a 1.9 to $12.9 \mathrm{~km} / \mathrm{h}$ reduction in traffic speeds due to light rain and a 4.8 to $16.1 \mathrm{~km} / \mathrm{h}$ reduction in speed was reported due to heavy rain. Work by Rakha et al. studied the impact of inclement weather (rain, snow, and freezing rain) and visibility on the traffic stream fundamental diagram using data from three cities: Minneapolis/St. Paul, Seattle, and Baltimore [16, 17]. The precipitation data included intensities up to 1.6 and $0.33 \mathrm{~cm} / \mathrm{h}$ for rain and water equivalent of snow intensity, respectively. The study demonstrated that the traffic stream jam density is not affected by weather conditions. Snow results in larger reductions in traffic stream free-flow speed and capacity when compared to rain. Reductions in roadway capacity are not affected by the precipitation intensity except in the case of snow. Reductions in free-flow speed and speed-at-capacity increase as the rain and snow intensities increase. Finally, the paper also developed free-flow speed, speed-at-capacity, and capacity weather adjustment factors that are multiplied by the base clear condition variables to compute inclement weather parameters. These adjustment factors vary as a function of the precipitation type, precipitation intensity, and visibility level [18]. These models were used to develop a macroscopic weather-tuned perimeter controller that was implemented in the Integration software $[19,20]$ and tuned using clear weather data and then tested for clear and inclement weather conditions $[21,22]$. Alhassan and Ben-Edigbe studied the effect of precipitation on traffic flow and, thus, the consequences on highway capacity [9]. They found that there is a traffic flow contraction and speed reduction of $3.52 \%$ due to precipitation.
Similarly, Akin et al. established a relationship between speed and volume as a function of weather conditions in freeway sections in Istanbul metropolitan area [23]. Results show that rain decreased the average speed by 8 to $12 \%$ while wet surface conditions reduced the average speeds by 6 to $7 \%$. Cools et al. focused on the effect of weather conditions on daily traffic intensities in Belgium [13], in which results also showed that rainfall reduces traffic intensity. However, Smith et al. argued, by analyzing data from the Hampton Roads of Virginia, that the reduction in speed due to rain was 3 to $5 \%$, which is not as dramatic as other studies found [24]. Rakha et al. had found earlier that the impact of precipitation type (rain, snow, and freezing rain) and visibility was different depending on the location and the level of familiarity of drivers to the weather conditions [17]. Specifically, Rakha et al. demonstrated that drivers in cities where rain and snow are more common seemed to exhibit larger reductions in speeds.

Figure 1 portrays the theoretical framework of the interactions between rain, incidents, traffic speed, and traffic flow $[13,25]$. An example of how this framework would run is the case of a heavy precipitation condition at a highway. As soon as the rain starts, drivers might begin abruptly or gradually brake to reduce their travel speed. This reaction could create a shockwave as a byproduct of the transitioning from free-flow speed into congestion, which reduces road capacities and corresponding traffic flow. Incidents may occur during this transition such as road accidents, increasing its frequency and/or its severity. This example shows that road incidents, rain, traffic speed, and flow are the consequence of an interplay of a multifactorial process, including behavioral, environmental, and technological factors $[26,27]$.

Most of the previous studies modeled traffic speed with a fixed mean albeit the two different regimes that it can correspond to (i.e., flow-free and congested). This study attempts to quantify the impacts of precipitation and incidents on traffic stream speed along a section of Interstate 64 (I-64) in Virginia. We modeled the speed using a mixture of two components of linear regression. The first component represents the congested regime and the second component represents the free-flow regime, in which the means of each component vary as a function of the precipitation level and lane closure ratio. The fitted model allows us to understand how the average speed (centroid) of each component (cluster) changes as a function of precipitation and incident severity. Understanding this should help transportation planners, operation traffic engineers, and policymakers to determine the extent of the precipitation and incidents' effect when conducting level-of-service analysis. It also could ease financial and time loss by traffic incidents, by providing effective rerouting and evacuation plans for emergency vehicles, providing drivers with real-time advisory messages to effectively avoid impacted areas, or even for long-term policymaking.

\section{Dataset}

In general, three datasets were used in this study, namely, INRIX traffic data from 2013 to 2016 along a section of 


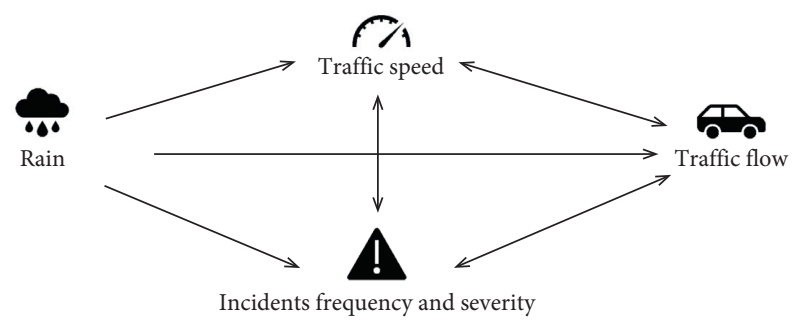

FIGURE 1: Relationship between rain, incidents, traffic speed, and traffic flow.

Interstate 64 (I-64), weather data, and incident data. The selected corridor data was used to obtain a daily traffic speed matrix over spatial and temporal domains. The precipitation data was collected at the weather stations closest to the TMC station based on the ZIP codes. And the incidents were assigned to the corresponding TMC and were translated into a matrix with dimensions identical to the speed data dimensions.

3.1. Study Area. INRIX traffic data from the years of 2013 to 2016 along the eastbound section of Interstate 64 (I-64) in the State of Virginia were used in this study. The selected I-64 freeway corridor, which is shown in Figure 2, includes 30 segments along 25.6 miles, and the length of each segment is unevenly divided in the raw data. Travel times for each roadway segment were included in the raw data, which were collected at $5 \mathrm{~min}$ intervals to reduce the stochastic noise and measurement error and obtain a daily traffic speed matrix over spatial (upstream to downstream) and temporal (from 0:00 a.m. to 23:55 p.m.) domains.

3.2. Data Reduction. Data reduction was performed to transfer the raw measured data into the required input data format. In general, the spatiotemporal traffic speed matrix is the fundamental format used as for the input data. Thus, reduction of INRIX probe data is one example where the spatiotemporal traffic speed matrix is used, and a similar process can be applied to other types of measured data (e.g., loop detector). INRIX data, which was mainly collected by GPS-equipped vehicles, supplemented with traditional road sensor data, along with mobile devices and other sources, were provided for each roadway segment and time interval. Each roadway segment corresponds to a traffic message channel (TMC) station, in which the geographic TMC station information is also provided. The average travel time for each TMC station can be used to derive the spatiotemporal traffic state matrix. However, raw INRIX data also includes missing data. As a result, the data reduction and preprocessing were performed to address these problems as Figure 3 depicts.

Based on the geographic information of each TMC station, raw data were sorted along the roadway direction (i.e., eastbound and westbound). Afterwards, the space mean speed was calculated by dividing the length of the roadway covered by a TMC by the average travel time. The raw INRIX data used in this study were aggregated at 5 min time intervals into a matrix with two dimensions: spatial and temporal intervals. Data imputation methods were developed to estimate the missing data using the neighboring cell values. As shown in Figure 4, the result of this process is a daily spatiotemporal traffic state matrix, which can be used for clustering.

3.3. Weather Data. In addition to the traffic data, precipitation data were collected at the weather stations closest to the TMC station based on the provided ZIP codes, as shown in Table 1. The precipitation data were translated into data matrices with dimensions identical to those of the speed data in order to study the impact of the weather (i.e., precipitation) on the highway traffic speed. Each roadway segment corresponds to a traffic message channel (TMC) station. The entire road segment is covered by four weather stations, as shown in Table 1. Each of these weather stations covers a set of ZIP codes. Consequently, there is only one station that covers one TMC. In other terms, the TMC is mapped to the weather station that matches the TMC's ZIP code. The weather data is shaped as a daily spatiotemporal weather matrix that has the same size as the daily spatiotemporal traffic state matrix (i.e., weather data is aggregated at $5 \mathrm{~min}$ time intervals), in which we used the mean to aggregate the precipitation.

3.4. Incident Data. The provided incident data was not assigned to a corresponding TMC, so we calculated the distance of the collected events using the events start latitude/longitude and end latitude/longitude. If the start and end coordinates were not the same (i.e., the distance was greater than zero), we calculated the midway point coordinates using the Vincenty method [28]. To assign a TMC to an event, we calculated the distance between the event's midway point coordinates and the corresponding TMC segment's start and end point coordinates. If the summation of both distances was greater than the TMC's segment length (with 5\% tolerance), then we assumed that the event did not belong to that TMC. We repeated the previous steps for all the TMC segments. Using the assigned TMC and the incident date and duration, the incident data were structured as data matrices with dimensions identical to the speed data dimensions. The resulted matrix was then used to investigate the impact of the incident on the highway traffic speed. Figure 5 shows a descriptive analysis for the incident data.

\section{Methods}

This study investigates the rain and incident impacts on the mean of highway traffic speed for different traffic regimes (i.e., congested and free-flow). A mathematical modeling technique was developed to pool rain and incident data and estimate the change of centroids without sorting the data into time bins. We developed a speed model to feature a mix of two linear regressions for the two traffic regimes. Each linear equation describes a relationship between two independent variables derived from the weather and incident data (i.e., precipitation level and ratio of closed 


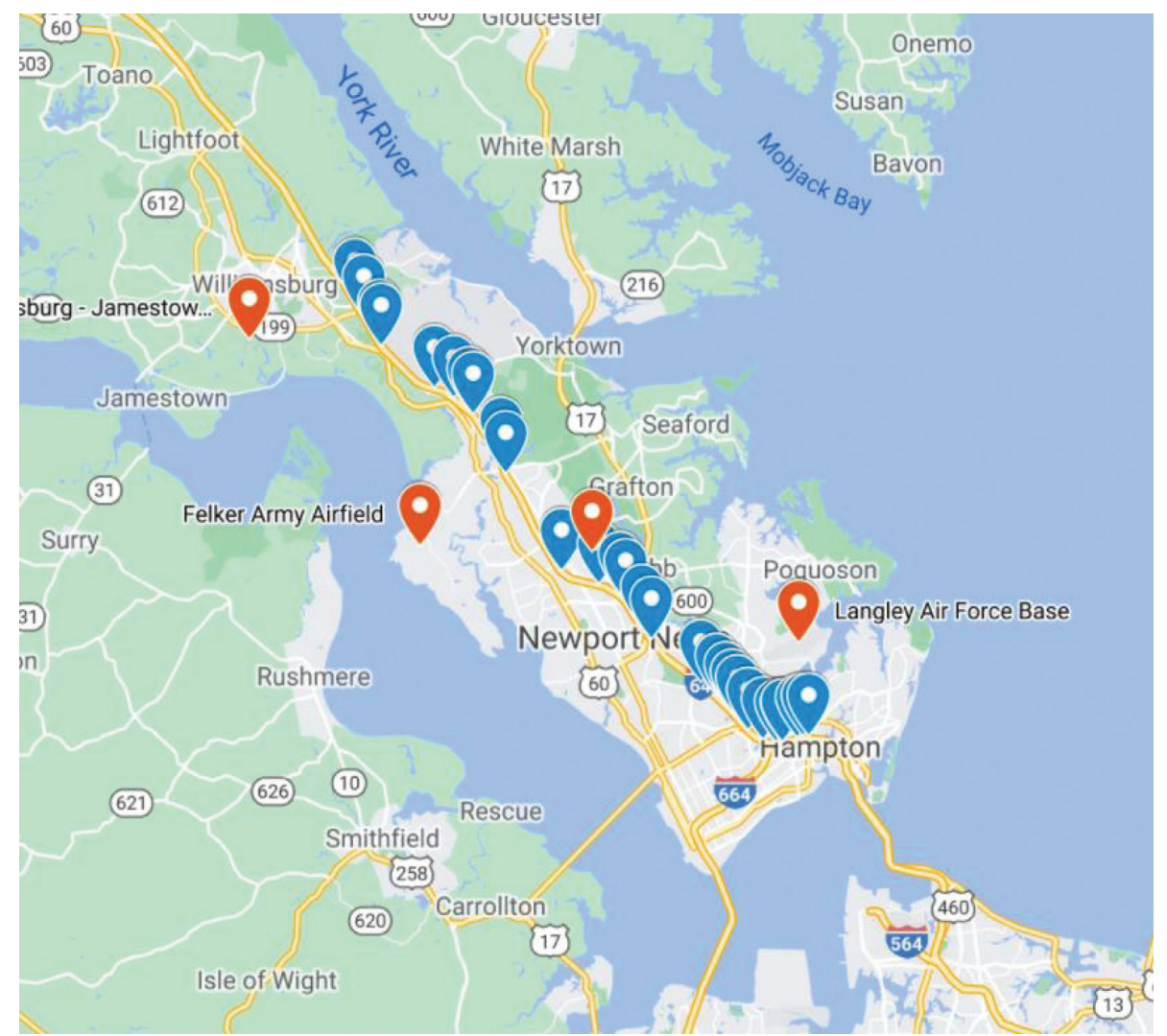

Figure 2: Layout of the study area on I-64, VA, where the blue markers show the start location of the TMCs and the orange markers show the location of the weather stations (source: Google maps).

lanes) and the dependent variable (i.e., traffic speed). To put differently, instead of embedding two components as regressors in one rigid model (i.e., with fixed means), the proposed speed model mixes the two components in a model whose means are function of speed when a segment of the road is congested and when it could be considered as free-flow speed.

4.1. Mixture of Linear Regressions (MLR). Finite Mixture of Linear Regressions (MLR) models are effective tools for investigating a wide variety of stochastic phenomena, in which they are used in many fields, including agriculture, biology, economics, medicine, and genetics $[29,30]$. In this study, it can be used to analyze the effect of rain and incidents on the traffic speed for different traffic regimes. Using mixture models attempts to mainly relax three limitations [8]. First, the mean of each component is not modeled as a function of the available predictors. Second, the proportion variable is fixed at each time bin, which limits the model's flexibility. Third, information provided given the time bin of the day is the probability of each component (fixed) and the 90th percentile. The following is a discussion of the background of the proposed model. Mixture of linear regressions (MLR) is one of the mixture families studied carefully in the literature $[31,32]$.

The mixture of linear regressions can be formulated as

$$
p(y \mid X)=\sum_{j=1}^{m} \frac{\lambda_{j}}{\sigma_{j} \sqrt{2 \pi}} e^{-\left(\left(y-X^{T} \beta_{j}\right)^{2} / 2 \sigma_{j}^{2}\right)}
$$

which means, in a different way, that

$$
y_{i}=\left\{\begin{array}{c}
x_{i}^{T} \beta_{1}+\varepsilon_{i 1} \text { with probability } \lambda_{1}, \\
x_{i}^{T} \beta_{2}+\varepsilon_{i 2} \text { with probability } \lambda_{2}, \\
\vdots \\
x_{i}^{T} \beta_{m}+\varepsilon_{i m} \text { with probability } 1-\sum_{q=1}^{m-1} \lambda_{q},
\end{array}\right.
$$

where $y$ is a response corresponding to a predictor's vector $X^{T}, \beta_{j}$ is a vector of regression coefficients for the jth mixture component, $\lambda_{j}$ is a mixing probability of the jth mixture component, and $m$ is the number of components in a mixture model.

However, the model parameters $\psi=\left\{\beta_{1}, \beta_{2}\right.$, $\left.\ldots, \beta_{m}, \sigma_{1}^{2}, \sigma_{2}^{2}, \ldots, \sigma_{m}^{2}, \lambda_{1}, \lambda_{2}, \ldots, \lambda_{m}\right\}$ can be estimated by maximizing the log-likelihood using the Expectation-Maximization algorithm (EM), given a set of response predictor pairs $\left(y_{1}, x_{1}\right),\left(y_{2}, x_{2}\right), \ldots,\left(y_{n}, x_{n}\right)$.

4.2. Expectation-Maximization Algorithm (EM). The EM algorithm was found to be powerful in several previous studies to estimate the parameters of finite mixture models [7, 33-35]. The EM algorithm iteratively finds the maximum 


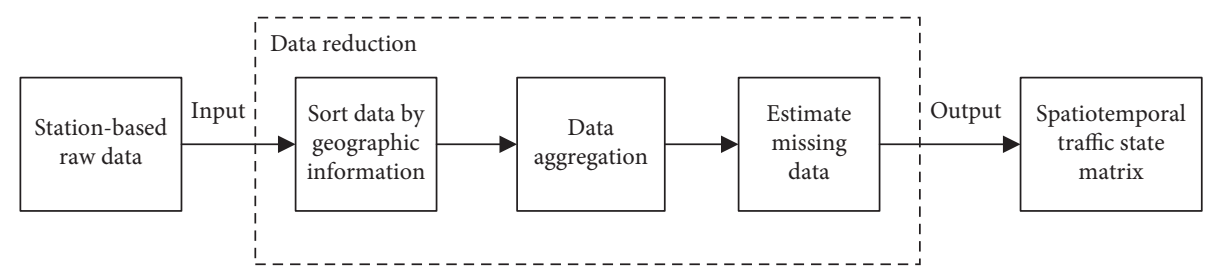

FIGURE 3: Data reduction of INRIX probe data.

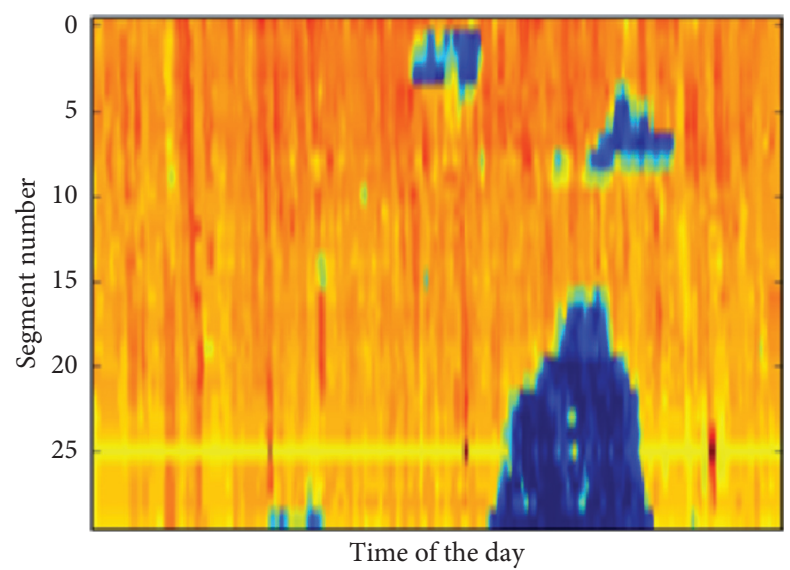

Figure 4: The spatiotemporal traffic speed matrix, where blue is the congested areas.

TABle 1: The TMC ZIP codes and the corresponding weather station.

\begin{tabular}{lc}
\hline Weather station & ZIP code \\
\hline Williamsburg-Jamestown Airport & 23185 \\
Felker Army Airfield & 23603 \\
Newport News/Williamsburg Intl. Airport & $23601,23602,23603,23606$, and 23608 \\
Langley Air Force Base Airport & 23666 and 23669 \\
\hline
\end{tabular}

likelihood estimates by alternating between two main steps, namely, the E-step and M-step. The equations of these two steps to estimate the model parameters are shown in Algorithm 1.

\section{Results}

To apply the proposed model, speed data corresponding to each TMC was divided by the posted limit speed at that TMC. Because speed distributions are skewed, the lognormal distribution is preferred to the normal distribution. Log of the relative speed is used as the response variable $(y)$. On the other hand, the incident predictor was defined as the number of lanes closed due to the incident divided by the facility's total number of lanes. A total of 750 realizations were drawn randomly from the data set to construct random samples. Each of which had the log of the relative speed-i.e., log (speed/posted speed) - as the response and precipitation level and percentage of lane closure as the explanatory predictors for the model. We fitted the mixture of two linear regressions (i.e., components) using this data. The model will be useful to describe the change of the mean of the speed distribution of the congested regime, as the first component, and free-flow regime, as the second component, as a function of the precipitation level and the percentage of lane closure.

The coefficients of the predictors $\left(\beta_{1}, \beta_{2}\right)$, the variances $\left(\sigma_{1}^{2}, \sigma_{2}^{2}\right)$, and the proportions $\left(\lambda_{1}, \lambda_{2}\right)$ corresponding to each component were estimated using the iterative EM algorithm (i)-(iii) in Algorithm 1 for each realization. The final model parameters are the mean of the 750 estimates, which was drawn randomly from the dataset. Once the final model is derived, the mean of the speed distribution varies with the precipitation intensity and road closure proportion for both components. Given any combination of explanatory predictors, the final model computes the mean speeds for the two components. Table 2 shows the estimated model parameters.

As Figure 6 depicts, the first component mean-in the congested regime-decreases as the percentage of road closure increases. The change in the mean of the first component with the precipitation seems to be insignificant with a relatively very trivial change in the mean as the precipitation level varies. However, in order to check the significance of the two explanatory predictors, we examined 


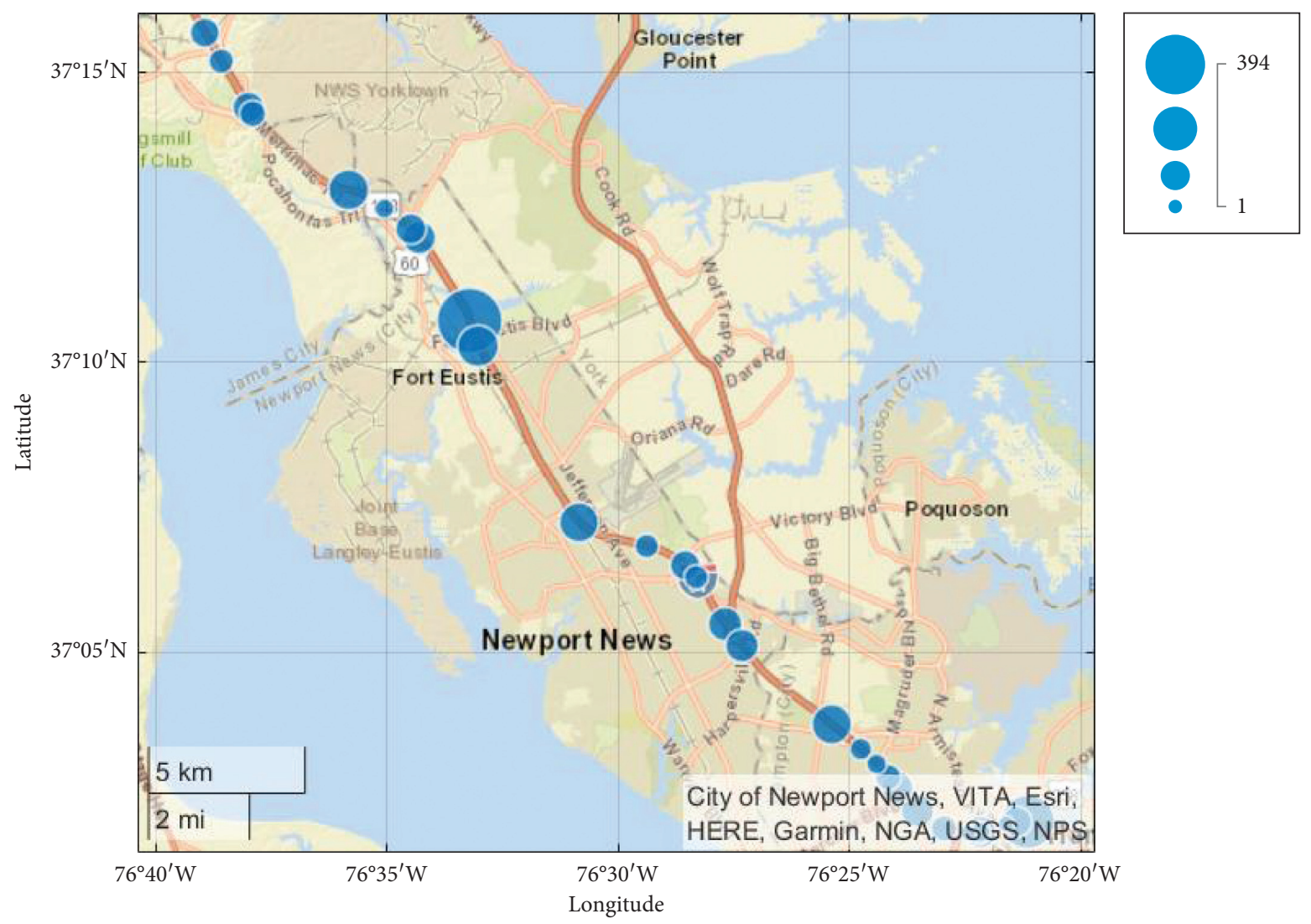

(a)

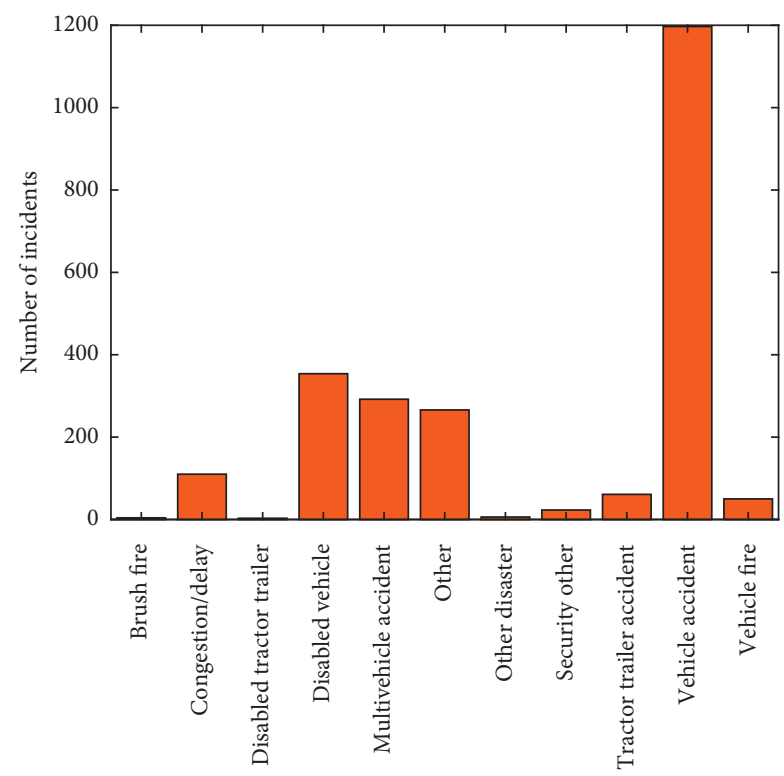

(b)

Figure 5: Continued. 


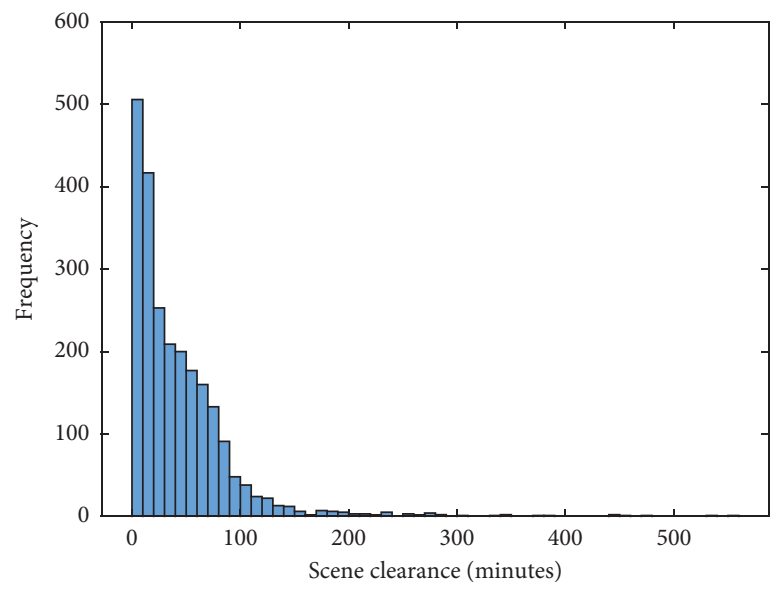

(c)

Figure 5: Above: map of the study area on I-64. VA shows the location and the number of incidents at each TMC (source: Google maps). Below left: histogram shows the categories of the reported incidents. Below right: histogram shows the distribution of the scene clearance times for all the 2365 incidents, which are the total during the analysis period.

Let $\psi^{(k)}$ be parameter estimates after the kth iteration.

E-step:

(1) Estimate the posterior probability of the ith observation comes from component $j$,

(i) $w_{i j}^{(k+1)}=\left(\lambda_{j}^{(k)} \phi_{j}\left(y_{i} \mid x_{i}, \psi^{(k)}\right) / \sum_{j=1}^{m} \lambda_{j}^{(k)} \phi_{j}\left(y_{i} \mid x_{i}, \psi^{(k)}\right)\right)$

where $\phi_{j}\left(y_{i} \mid x_{i}, \psi^{(k)}\right)$ is the probability density function of the jth component.

M-step:

(2) Find a new parameter that estimates $\psi^{(k+1)}$ by maximizing the log-likelihood function in equation

$(p(y \mid X))=\sum_{i=1}^{n}\left(\lambda_{j} / \sigma_{j} \sqrt{2 \pi}\right) e^{-\left(\left(y-X^{T} \beta_{j}\right)^{2} / 2 \sigma_{j}^{2}\right)}$

(ii) $\lambda_{j}^{(k+1)}=\left(\sum_{i=1}^{n} w_{i j}^{(k+1)} / n\right)$ and

(1) is calculated as follows:

(iii) $\widehat{\beta}_{j}^{(k+1)}=\left(X^{T} W_{j} X\right)^{-1} X^{T} W_{j} Y$ where $X$ is an $n x(p+1)$ predictor matrix, $\mathrm{Y}$ is the corresponding $\mathrm{nx} 1$ response vector, and $\mathrm{W}$ is an nxn diagonal matrix. Now, by having $w_{i j}^{(k+1)}$ along its diagonal:

(iv) $\widehat{\sigma}_{j}^{2(k+1)}=\left(\left(\sum_{i=1}^{n} w_{i j}^{(k+1)}\left(y_{i}-x_{i}^{T} \beta_{j}^{(k+1)}\right)^{2}\right) /\left(\sum_{i=1}^{n} w_{i j}^{(k+1)}\right)\right)$

(3) Alternate repeatedly between the E-step and M-step until the incomplete log-likelihood converges to an arbitrarily small value as follows:

(v) $\left|\prod_{i=1}^{n} \sum_{j=1}^{m} \lambda_{j}^{(k+1)} \phi_{j}\left(y_{i} \mid x_{i}, \psi^{(k+1)}\right)-\prod_{i=1}^{n} \sum_{j=1}^{m} \lambda_{j}^{(k)} \phi_{j}\left(y_{i} \mid x_{i}, \psi^{(k)}\right)\right|<\xi$ where $\xi$ is a small number.

Algorithm 1: The EM algorithm.

the histogram of the estimated coefficients of the 750 random samples, shown in Figure 7(a). We found that roughly $20 \%$ of the estimates are less than zero, indicating that the 95\% confidence interval contains zero. This confirms that precipitation level does not significantly influence the mean of the first component. By the same token, we examined the histogram of the closure proportion, shown in Figure 7(b), and found that the $95 \%$ confidence interval does not contain zero, leading us to conclude that the closure proportion significantly reduces the mean of the first component.

On the other hand, Figure 8 depicts that the second component (free-flow regime) mean decreases as the ratio of closed lanes increases or as the precipitation level increases. To test the significance of the two explanatory predictors, we examined the histogram of the estimated coefficients of 750 random samples, shown in Figure 9. Neither coefficients' histograms include zero. Consequently, their 95\% confidence intervals do not contain zero, and we conclude that as the precipitation increases or the closed lanes ratio increases, the mean speed of the second component decreases.

5.1. Cut-Off Speed. We used the estimated parameters of the closed lanes ratio $\left(\sigma_{1}^{2}, \sigma_{2}^{2}, \lambda_{1}, \lambda_{2}\right)$ of the model and the Bayes rule to compute the cut-off relative speed such that the misclassification error is minimized. This cut-off speed relative to the closed lanes ratio is used as a classification threshold between the congested and the free-flow regimes. 
TABLE 2: Parameters' estimates of the mixture of log-normal regression model.

\begin{tabular}{lcc}
\hline Parameter & First component (congested regime) & Second component (free-flow regime) \\
\hline Intercept & -0.5802 & 0.0860 \\
Precipitation coefficient & 0.0480 & -0.1250 \\
Ratio of closed lanes coefficient & -1.0075 & -0.0762 \\
Standard deviation & 0.6544 & 0.0636 \\
Mixing probability $(\lambda)$ & 0.0987 & 0.9013 \\
\hline
\end{tabular}

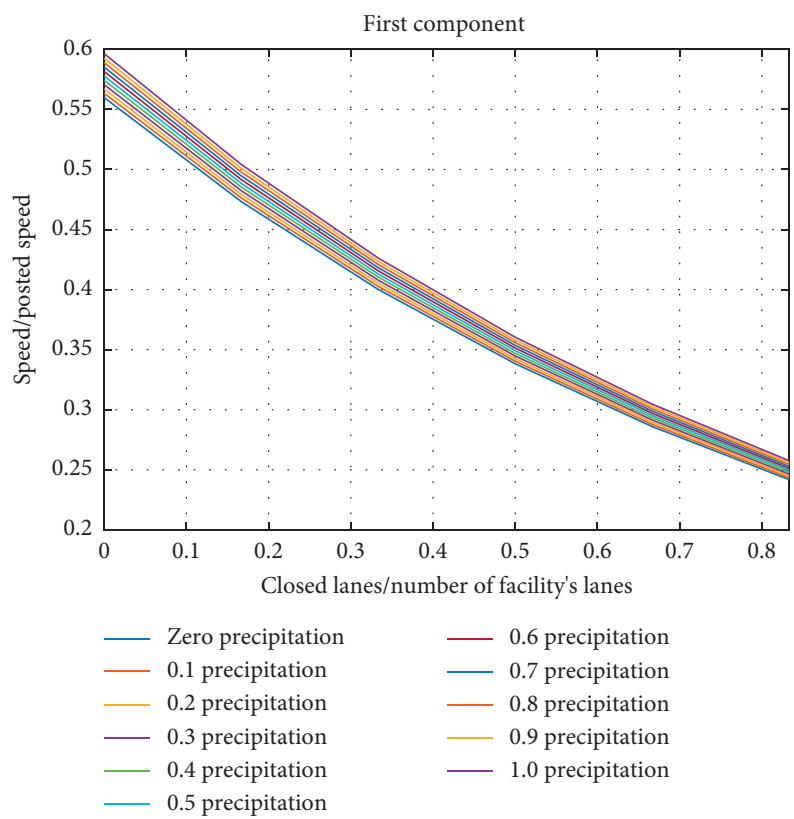

FIgURE 6: The change of the mean of the first component (congested regime) with the precipitation intensity and proportion of closed lanes.

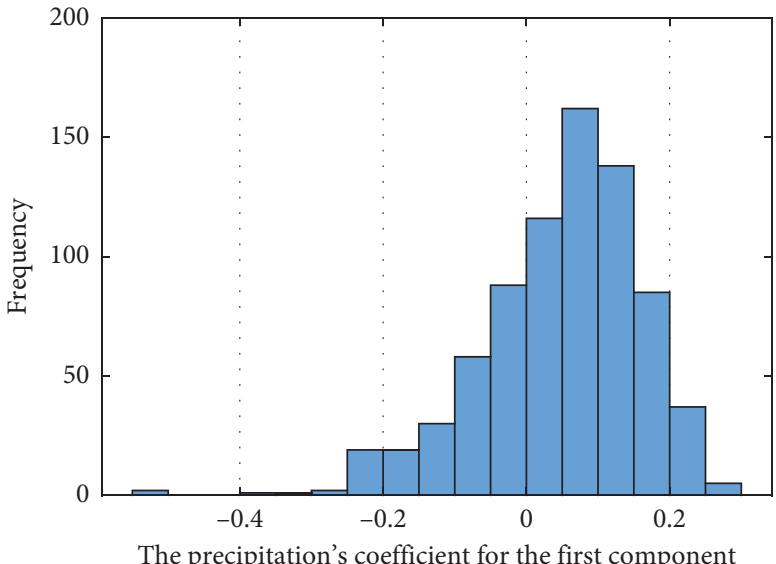

(a)

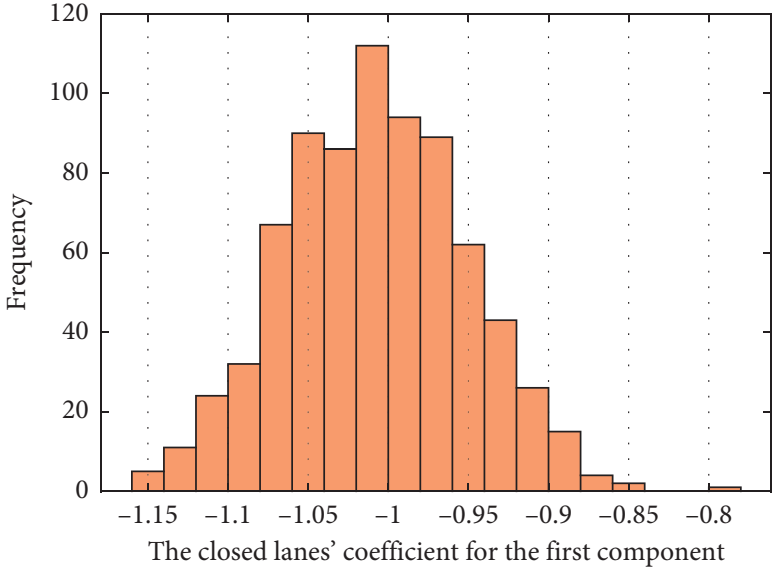

(b)

FIgURE 7: Histogram of the precipitation (a) and closed lanes ratio (b) coefficient for the first component (congested regime).

The output of applying the above threshold is a spatiotemporal binary matrix with dimensions identical to the spatiotemporal speed matrix. A "1" in the binary matrix identifies a segment as congested, and a " 0 " represents free-flow conditions. Given the probability distribution for congestion and free-flow speeds, the Bayes rule is used as shown in 


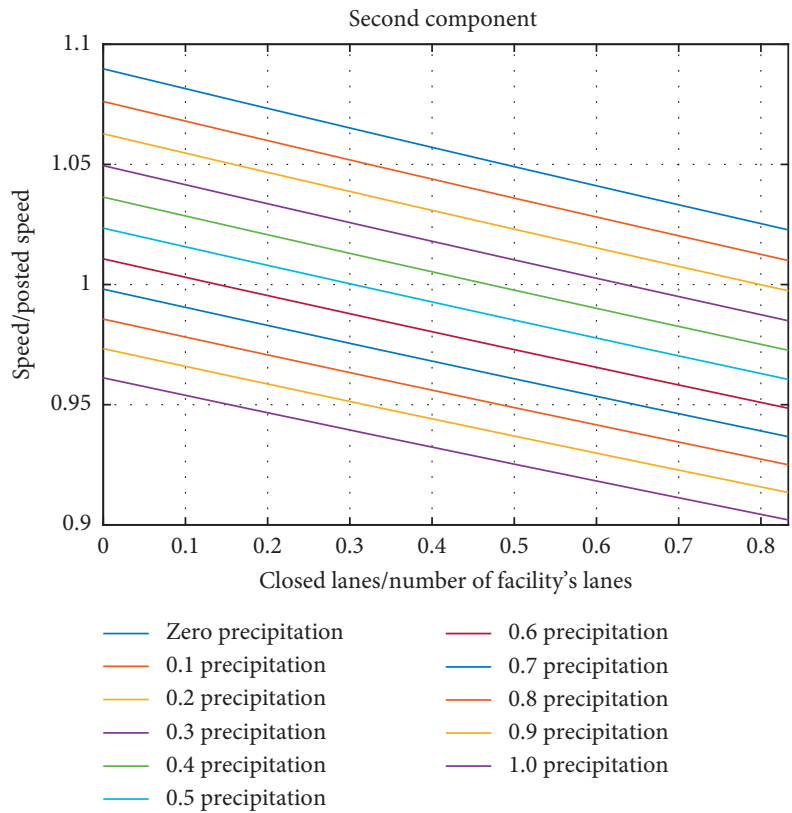

Figure 8: The change of the mean of the second component (free-flow regime) with the precipitation and ratio of closed lanes.

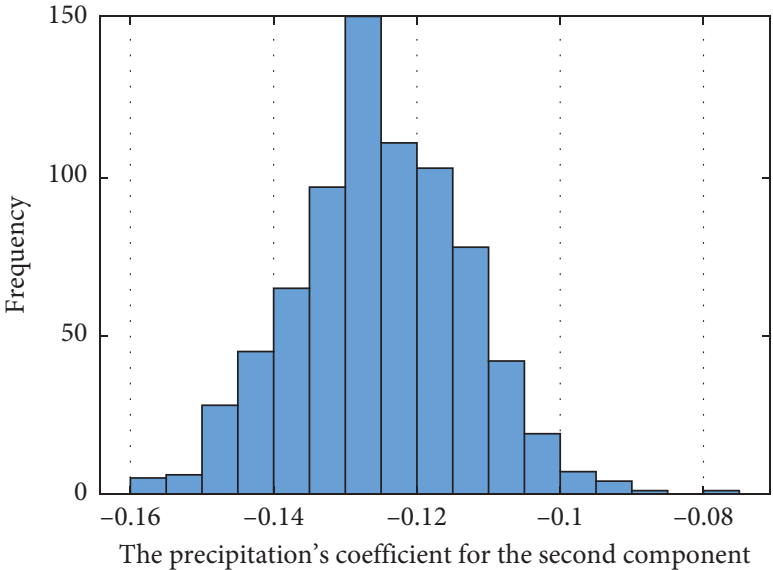

(a)

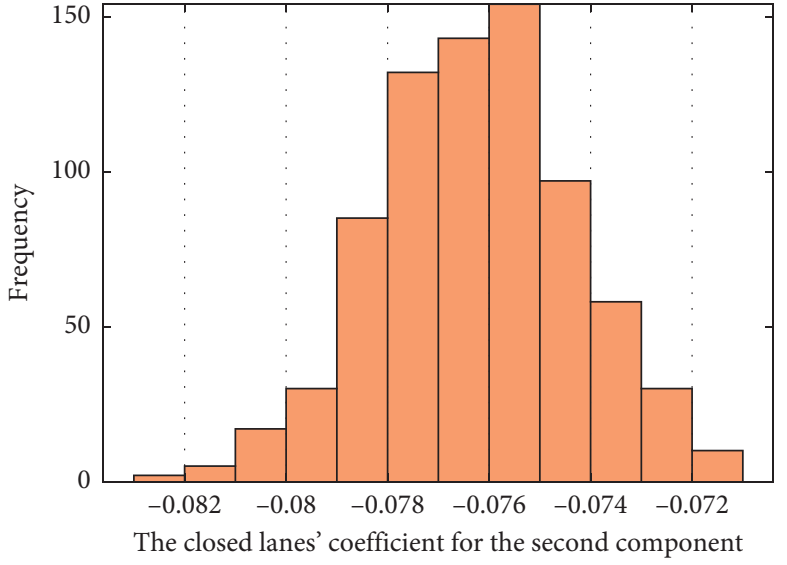

(b)

Figure 9: Histogram of the precipitation (a) and closed lanes ratio (b) coefficient for the second component (free-flow regime).

$$
\begin{gathered}
p\left(v_{c} \mid v_{i}\right)=p\left(v_{i} \mid v_{c}\right) p\left(v_{c}\right), \\
p\left(v_{f} \mid v_{i}\right)=p\left(v_{i} \mid v_{f}\right) p\left(v_{f}\right),
\end{gathered}
$$

where $v_{i}$ is the speed at any time $i, v_{c}$ is the speed at congestion, and $v_{f}$ is the speed at free-flow. The optimum threshold is the value of the speed at which $p\left(v_{c} \mid v_{i}\right)=p\left(v_{f} \mid v_{i}\right)$, as illustrated in Figure 10(a). Assuming this threshold can be calculated, any speed above the threshold line is classified as free-flow and any speed below it is identified as congested. The relative cut-off speed for the I-64 stretch studied in this paper is shown in Figure 10(b). All segments with relative speeds greater than the threshold are classified as free-flow segments, and other segments are classified as congested segments. For example, if half of a segment on I-64 is closed (one closed lane out of two available) and the observed speed is greater than $\sim 0.54$ of the speed limit, then the segment is included in the free-flow regime. Otherwise, the segment is considered in the congested regime.

\section{Discussion}

This study aims not only to understand the effect of incidents and precipitation, each separately, but also to identify the relationship between the two factors. This comprehensive analysis allows policymakers to assess the appropriateness and effectiveness of the adapted local traffic management strategies. Table 3 shows the resulting reduction in the traffic speed due to different levels of precipitation and incident 


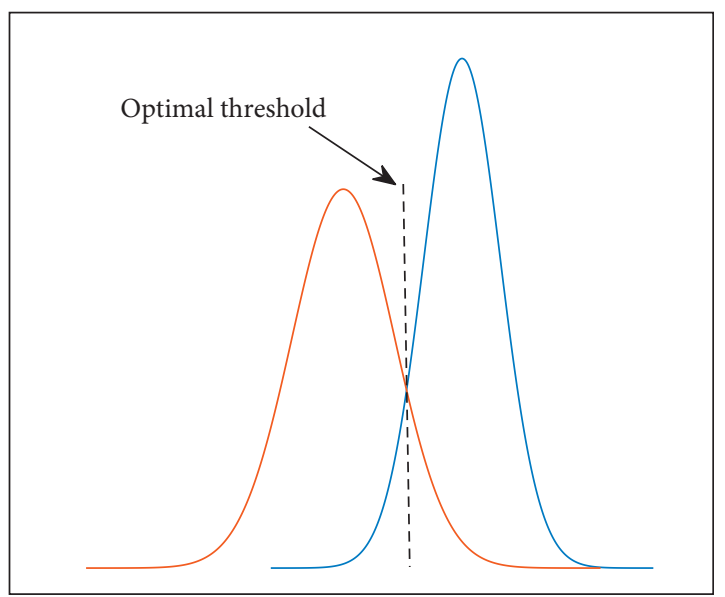

- Free-flow component

— Congested component

(a)

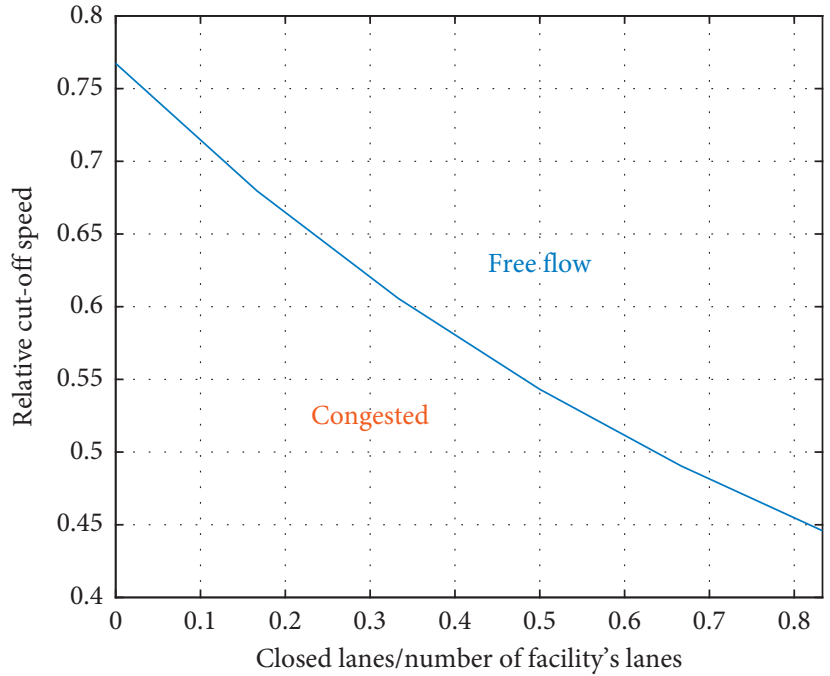

(b)

FIGURE 10: The Bayesian approach (a) and the resulting threshold cut-off speeds for the studied section (b).

TABLE 3: Reduction in traffic stream speed due to precipitation and incidents in the free-flow regime and only due to incidents in the congested regime.

\begin{tabular}{lcc}
\hline Incident severity & Precipitation level & Reduction in traffic speed (\%) \\
Congested regime
\end{tabular}

severity when the observed speed is in the free-flow or congested regimes along the I-64 section of highway in Virginia. To define precipitation level, we assumed that if precipitation is less than half the average precipitation data that were collected at the weather stations during the study period (2013-2016), then it is light rain; otherwise, it is a heavy rain. To define the incident severity, we assumed that if a third (or less) of the lanes are blocked, then it is low severity. However, if the closed lanes are more than a third, then this is high severity incident. The logic of this assumption is based on the fact that the studied segment of I-64 has either two or three lanes in the eastbound direction, which means that if only one lane is closed, we considered this a low severity incident. By the same token, if two lanes or three are blocked, this is considered as a high severity incident. Our analysis also shows that precipitation is a statistically insignificant factor when speed is congested (see Figure 7), so we only reported the effect of different incident severities on the traffic stream speed when the road is congested in Table 3. Results of the free-flow regime show that, without taking the incident impact into account, while light rain has an insignificant effect on the traffic stream speed, heavy rain slightly reduces the speed by a maximum of $4 \%$. This resulted in an undramatic precipitation effect, which coincides with the conclusion of a previous study on the same region [24]. However, when considering incidents, light rain has a marginal effect on the speed when the incident severity is low but reduces the speed by $0.6-4 \%$ when the incident severity is high. In turn, heavy rain reduces the speed by $0.6-6.5 \%$ when the incident severity is low but has roughly double this effect when the incident severity is high. In contrast, whereas precipitation has an insignificant effect on the traffic stream speed when the road is congested, low and high incident severity incidents have steep effects ranging from $41-57 \%$ and $58-75 \%$, respectively.

\section{Conclusion}

Several initiatives are being taken by Traffic Management Centers (TMCs), incident management agencies, and policymakers to mitigate congestion, improve incidentresponding time, and develop preplanned diversion and emergency routes. These initiatives include leveraging the use of Intelligent Transportation Systems (ITSs) and organizing effective coordination amongst different agencies. To meet these aggressive yet required goals, agencies should 
have a better understanding of the interplay of different factors that affect traffic stream speed. This study investigates the effect of precipitation and incidents on the speed of traffic on I-64 in Virginia. Light and heavy rain reduce the decision time to policymakers and lessen the amount of drivers' control on their vehicles by disturbing visibility, increasing physical discomfort, and reducing the roadway coefficient of friction. Road incidents increase the vulnerability as they could create traffic shockwaves, which reduce road capacities and corresponding traffic flow. To the best of our knowledge, this is the first study that discusses the relationship between precipitation and incidents as factors that would have a combined impact on traffic speed. Furthermore, this study reckons for the two different regimes that the traffic speed could be classified into, free-flow and congestion, using a mixture model of two linear regressions. Using mixture models provides the needed flexibility by having two components with two different means corresponding to the two different speed regimes.

This study used INRIX traffic data from 2013 to 2016 along a 25.6-mi section of Interstate 64 in Virginia. Precipitation data were collected from weather stations closest to the TMC station, and incident data were assigned to the corresponding TMC. Results show that the effect on traffic speed is vividly higher if the road has already been congested, yet the significant effect is derived only from one factor, namely, incident severity. Specifically, low and high severity incidents produce speed reductions ranging from $41 \%$ to $57 \%$ and $58 \%$ to $75 \%$, respectively. Regardless of whether the incident impact is considered, the effect of light rain in the free-flow regimes ranges from insignificant to a $4 \%$ speed reduction. On the other hand, the effect of heavy rain ranges from a $0.6 \%$ to a $6.5 \%$ speed reduction when the incident severity is low but has a roughly double effect when the incident severity is high.

\section{Data Availability}

Data were acquired by VDOT and FHWA from INRIX.

\section{Conflicts of Interest}

The authors declare no conflicts of interest regarding the publication of this paper.

\section{Acknowledgments}

This study was jointly funded by the Federal Highway Administration (FHWA) and the Virginia Department of Transportation (VDOT).

\section{References}

[1] R. Kala and K. Warwick, "Motion planning of autonomous vehicles in a non-autonomous vehicle environment without speed lanes," Engineering Applications of Artificial Intelligence, vol. 26, no. 5-6, pp. 1588-1601, 2013.

[2] S. Glaser, B. Vanholme, S. Mammar, D. Gruyer, and L. Nouveliere, "Maneuver-based trajectory planning for highly autonomous vehicles on real road with traffic and driver interaction," IEEE Transactions on Intelligent Transportation Systems, vol. 11, no. 3, pp. 589-606, 2010.

[3] L. Kalampoukas, Congestion Management in High Speed Networks, University of California, Santa Cruz, CA, USA, 1997.

[4] K. Boriboonsomsin, M. J. Barth, W. Zhu, and A. Vu, "Ecorouting navigation system based on multisource historical and real-time traffic information," IEEE Transactions on Intelligent Transportation Systems, vol. 13, no. 4, pp. 1694-1704, 2012.

[5] F. He, X. Yan, Y. Liu, and L. Ma, "A traffic congestion assessment method for urban road networks based on speed performance index," Procedia Engineering, vol. 137, pp. 425-433, 2016.

[6] F. Li, Y. Yu, H. Lin, and W. Min, "Public bus arrival time prediction based on traffic information management system," in Proceedings of the 2011 IEEE International Conference on Service Operations, Logistics and Informatics, pp. 336-341, Beijing, China, July 2011.

[7] M. Elhenawy, A. A. Hassan, and H. A. Rakha, "Travel time modeling using spatiotemporal speed variation and a mixture of linear regressions," in Proceedings of the 4th International Conference on Vehicle Technology and Intelligent Transport Systems, pp. 113-120, Funchal, Portugal, 2018.

[8] F. Guo, Q. Li, and H. Rakha, "Multistate travel time reliability models with skewed component distributions," Transportation Research Record: Journal of the Transportation Research Board, vol. 2315, no. 1, pp. 47-53, 2012.

[9] H. M. Alhassan and J. Ben-Edigbe, "Highway capacity prediction in adverse weather," Journal of Applied Sciences, vol. 11, no. 12, pp. 2193-2199, 2011.

[10] U.S. DOT Federal Highway Administration, FHWA Operations-Operations Story, Washington, DC, USA, 2020, http://www.ops.fhwa.dot.gov/aboutus/opstory.htm.

[11] T. H. Maze, M. Agarwal, and G. Burchett, "Whether weather matters to traffic demand, traffic safety, and traffic operations and flow," Transportation Research Record: Journal of the Transportation Research Board, vol. 1948, pp. 170-176, 2006.

[12] A. T. Ibrahim and F. L. Hall, Effect of Adverse Weather Conditions on Speed-Flow-Occupancy Relationships, Transportation Research Board, Washington, DC, USA, 1994.

[13] M. Cools, E. Moons, and G. Wets, "Assessing the impact of weather on traffic intensity," Weather, Climate, and Society, vol. 2, no. 1, pp. 60-68, 2010.

[14] H. J. Haule, T. Sando, R. Lentz, C.-H. Chuan, and P. Alluri, "Evaluating the impact and clearance duration of freeway incidents," International Journal of Transportation Science and Technology, vol. 8, no. 1, pp. 13-24, 2019.

[15] H. Rakha, M. Farzaneh, M. Arafeh, R. Hranac, E. Sterzin, and D. Krechmer, Empirical Studies on Traffic Flow in Inclement Weather, Virginia Tech Transportation Institute, Blacksburg, VA, USA, 2007.

[16] H. Rakha, M. Farzaneh, M. Arafeh, and E. Sterzin, "Inclement weather impacts on freeway traffic stream behavior," in Proceedings of the Transportation Research Board 87th Annual Meeting, Washington, DC, USA, January 2008.

[17] H. Rakha, M. Farzaneh, M. Arafeh, and E. Sterzin, "Inclement weather impacts on freeway traffic stream behavior," Transportation Research Record: Journal of the Transportation Research Board, vol. 2071, pp. 8-18, 2008.

[18] H. Rakha, M. Arafeh, and S. Park, "Modeling inclement weather impacts on traffic stream behavior," International Journal of Transportation Science and Technology, vol. 1, no. 1, pp. 25-47, 2012. 
[19] H. A. Rakha and M. Van Aerde, "Integration (c) Release 2.40 For windows: user's guide-volume I: fundamental model features," Virginia Tech, Blacksburg, VA, USA, 2020.

[20] H. A. Rakha and M. Van Aerde, "Integration @ release 2.40 for windows: user's guide - volume II: advanced model features," Virginia Tech, Blacksburg, VA, USA, 2020.

[21] M. Elouni and H. A. Rakha, "Weather-tuned network perimeter control - a network fundamental diagram feedback controller approach," in Proceedings of the 4th International Conference on Vehicle Technology and Intelligent Transport Systems (VEHITS), Funchal, Portugal, 2018.

[22] M. Elouni, H. A. Rakha, and Y. Bichiou, "Implementation and investigation of a weather- and jam density-tuned network perimeter controller," in Smart Cities, Green Technologies, and Intelligent Transport SystemsSpringer, Berlin, Germany, 2019.

[23] D. Akin, V. P. Sisiopiku, and A. Skabardonis, "Impacts of weather on traffic flow characteristics of urban freeways in Istanbul," Procedia - Social and Behavioral Sciences, vol. 16, pp. 89-99, 2011.

[24] B. L. Smith, K. G. Byrne, R. B. Copperman, S. M. Hennessy, and N. J. Goodall, "An investigation into the impact of rainfall on freeway traffic flow," in Proceedings of the 83rd Annual Meeting of the Transportation Research Board, Washington, DC, USA, 2004.

[25] M. J. Koetse and P. Rietveld, "Climate change, adverse weather conditions, and transport: a literature survey," in Proceedings of the 9th NECTAR Conference, Cambridge, UK, 2007.

[26] J. B. Edwards, "Weather-related road accidents in England and Wales: a spatial analysis," Journal of Transport Geography, vol. 4, no. 3, pp. 201-212, 1996.

[27] N. Levine, K. E. Kim, and L. H. Nitz, "Daily fluctuations in Honolulu motor vehicle accidents," Accident Analysis \& Prevention, vol. 27, no. 6, pp. 785-796, 1995.

[28] T. Vincenty, "Direct and inverse solutions of geodesics on the ellipsoid with application of nested equations," Survey Review, vol. 23, no. 176, pp. 88-93, 1975.

[29] A. T. Chaganty and P. Liang, "Spectral experts for estimating mixtures of linear regressions," in Proceedings of the 30th International Conference on Machine Learning, pp. 10401048, Atlanta, GA, USA, June 2013.

[30] K. Viele and B. Tong, "Modeling with mixtures of linear regressions," Statistics and Computing, vol. 12, no. 4, pp. 315-330, 2002.

[31] R. D. De Veaux, "Mixtures of linear regressions," Computational Statistics \& Data Analysis, vol. 8, no. 3, pp. 227-245, 1989.

[32] S. Faria and G. Soromenho, "Fitting mixtures of linear regressions," Journal of Statistical Computation and Simulation, vol. 80, no. 2, pp. 201-225, 2009.

[33] J. Kim and H. S. Mahmassani, "A finite mixture model of vehicle-to-vehicle and day-to-day variability of traffic network travel times," Transportation Research Part C: Emerging Technologies, vol. 46, pp. 83-97, 2014.

[34] S.-C. Lo, "Expectation-maximization based algorithm for pattern recognition in traffic speed distribution," Mathematical and Computer Modelling, vol. 58, no. 1-2, pp. 449-456, 2013.

[35] N. Wan, G. Gomes, A. Vahidi, and R. Horowitz, "Prediction on travel-time distribution for freeways using online expectation maximization algorithm," in Proceedings of the Transportation Research Board 93rd Annual Meeting, Washington, DC, USA, January 2014. 